\title{
CCD DEVELOPMENT ACTIVITIES AT ESO
}

O. Iwert

European Southern Observatory

\begin{abstract}
Advanced CCD detectors are the backbone of modern astronomical instrumentation working in the $300-1000 \mathrm{~nm}$ spectral range. The status and results of the different projects to design, manufacture and implement new CCD detectors for the ESO VLT instrumentation are presented. Emphasis is put into ESO's development activities together with industry's, and into first measurement results of thinned arrays in large formats obtained in 1994. In addition some recent cryostat developments are shown to illustrate the different requirements of VLT instruments in this area.
\end{abstract}




\section{DISCUSSION}

JORDEN: Could you discuss the merits of using an IR microscope for mosaic CCD alignment, compared with projecting images and reading out the devices?

IWERT: Projecting images and reading out CCDs: The complete chain of hardware and software (dewar, controller) is needed to read out the devices. It can only be done at room temperature as the devices cannot be easily manipulated when cold. The optical projection must be very accurate, so dedicated optics and mechanics is needed. It can only be tested with working CCDs, whereas it might be interesting to set the mosaic assembly mechanics cold with utilized mechanical samples. Using the infrared microscope: One can see right at the CCDs how the adjustment works and is not stacked by the above listed "obstacles".

FLORENTIN-NIELSON: The impressive QE figures you gave for the Lesser device (in UV) was that after UV flooding? Do you intend to reflood your CCD or keep it cold at all times?

IWERT: Yes, they were obtained after UV flooding. The long term stability of the QE after UV flooding has to be measured. In theory it should be sufficient to keep the CCD cold all the time after flooding.

BESSELL: What anti-reflection coatings have you discussed with Thomson?

IWERT: There is currently no definitive decision about the exact planned anti-reflection coating. However there will be definitely one deposited on the CCD. 